\title{
THE GLUING OF MAXIMAL IDEALS- SPECTRUM OF A NOETHERIAN RING-GOING UP AND GOING DOWN IN POLYNOMIAL RINGS
}

\author{
BY
}

\author{
ADA MARIA DE SOUZA DOERING AND YVES LEQUAIN
}

\begin{abstract}
If $M_{1}, \ldots, M_{s}$ are maximal ideals of a ring $R$ that have isomorphic residue fields, then they can be "glued" in the sense that a subring $D$ of $R$ with $R$ is integral over $D$ and $M_{1} \cap D=\ldots=M_{s} \cap D$ can be constructed.

We use this gluing process to prove the following result: Given any finite ordered set $\mathscr{B}$, there exists a reduced Noetherian ring $B$ and an embedding $\psi: \mathscr{B} \rightarrow \operatorname{Spec} B$ such that $\psi$ establishes a bijection between the maximal (respectively minimal) elements of $\mathscr{B}$ and the maximal (respectively minimal) prime ideals of $B$ and such that given any elements $\beta^{\prime}, \beta^{\prime \prime}$ of $\mathscr{B}$, there exists a saturated chain of prime ideals of length $r$ between $\psi\left(\beta^{\prime}\right)$ and $\psi\left(\beta^{\prime \prime}\right)$ if and only if there exists a saturated chain of length $r$ between $\beta^{\prime}$ and $\beta^{\prime \prime}$.

We also use the gluing process to construct a Noetherian domain $A$ with quotient field $L$ and a Noetherian domain $B$ between $A$ and $L$ such that: $A \hookrightarrow B$ possesses the Going Up and the Going Down properties, $A[X] \hookrightarrow B[X]$ is unibranched and $A[X] \hookrightarrow B[X]$ possesses neither the Going Up nor the Going Down properties.
\end{abstract}

1. Introduction and definitions. In [11], Nagata constructed his famous example of a local domain $D$ that does not satisfy the altitude formula. The basic idea of the construction is to consider two maximal ideals $M_{1}$ and $M_{2}$ of different height of a domain $R$ and to "glue" them in the sense of constructing a subring $D$ of $R$ that satisfies the following two properties: $R$ is integral over $D$ and $M_{1} \cap D=M_{2} \cap$ $D$. Nagata noted that if $R$ contains a field $K$ that is a field of representatives for both $R_{M_{1}}$ and $R_{M_{2}}$, then $M_{1}$ and $M_{2}$ can indeed be glued by taking $D=K+$ $\left(M_{1} \cap M_{2}\right)$; he also noted that $D$ is Noetherian if $R$ is Noetherian. The main difficulty that Nagata encountered was to come up with a Noetherian domain $R$ having two maximal ideals $M_{1}$ and $M_{2}$ of different height on which his gluing process could be performed. It is clear that such a ring $R$ cannot be a ring of quotients of a finite $K$-algebra, and it is working in a very clever way with formal power series that Nagata constructed one.

After Nagata, many authors have used his gluing process to solve problems of various kinds. One of those authors, Heitmann, has shown in [5, Theorem 2.1] that, given any finite ordered set $\mathscr{B}$, there exists a Noetherian domain $B$ and an embedding $\psi: \mathscr{B} \rightarrow$ Spec $B$ such that $\psi\left(\beta^{\prime}\right) \subset \psi\left(\beta^{\prime \prime}\right)$ are consecutive prime ideals of $B$ if $\beta^{\prime}<\beta^{\prime \prime}$ are consecutive elements of $\mathscr{B}$. His proof is difficult and relies on

Received by the editors June 5, 1979 and, in revised form, August 28, 1979.

AMS (MOS) subject classifications (1970). Primary 13B20, 13B25, 13C15, $13 E 05$.

Key words and phrases. Gluing of maximal ideals, integral extension, altitude formula, finite ordered set, spectrum of a Noetherian ring, Going Up, Going Down, unibranchness, polynomial ring. 
delicate properties of integral extensions of rings of formal power series.

Motivated by the geometric operation of gluing points in an algebraic variety, we develop in $\$ 2$ of this paper a very natural process to glue maximal ideals of a ring; this process requires much less stringent conditions than Nagata's technique does in order to be performed: a finite number of maximal ideals of a ring $R$ can be glued if their residue fields are isomorphic; there is no need for $R$ to contain any field at all. This is important in the applications because, contrary to the rings needed by Nagata, such rings $R$ are very easy to obtain; for example, if $k$ is a field, if $t_{1}, \ldots, t_{n}, \ldots$ is an infinite set of indeterminates over $k$ and if $K=$ $k\left(t_{1}, \ldots, t_{n}, \ldots\right)$, then the ring $R=K\left[X, Y_{1}, Y_{2}\right]_{(X) \cup\left(Y_{1}, Y_{2}\right)}$ can already be used to produce a local domain $D$ that does not satisfy the altitude formula by gluing the maximal ideals $X R$ and $\left(Y_{1}, Y_{2}\right) R$ with our process. A property of our gluing process that is important for the applications we give in the following sections is that, roughly speaking, the prime ideals that are not being glued stay unaltered. Also, we note that it is possible to glue several finite families of maximal ideals of a ring $R$.

The constructions that have made use of Nagata's gluing process have been invariably lengthy and complicated. It will be apparent through the applications given in this paper that, requiring less rigid conditions in order to be performed, our gluing process allows an easier control of other data and, consequently, lighter constructions.

We first apply our gluing process in $\$ 3$ to prove the following result that is much stronger than the previously stated result of Heitmann: given any finite ordered set $\mathscr{B}$, there exists a reduced Noetherian ring $B$ and an embedding $\psi: \mathscr{B} \rightarrow \operatorname{Spec} B$ such that if $\beta^{\prime}, \beta^{\prime \prime}$ are any elements of $\mathscr{B}$, then there exists a saturated chain of prime ideals of length $r$ in $B$ between $\psi\left(\beta^{\prime}\right)$ and $\psi\left(\beta^{\prime \prime}\right)$ if and only if there exists a saturated chain of length $r$ in $\mathscr{B}$ between $\beta^{\prime}$ and $\beta^{\prime \prime}$. Since it is also possible to choose $B$ such that $\psi$ establishes a bijection between the maximal (respectively minimal) elements of $\mathscr{B}$ and the maximal (resp. minimal) prime ideals of $B$, we see that our result is the analogue for Noetherian rings of the following result for rings proved by Hochster [6, Proposition 10, p. 56], and independently by Lewis [7, Theorem 2.10, p. 428]: every finite ordered set is order isomorphic to Spec $D$ for some ring $D$. As a corollary we also show that given any integer $n \geqslant 2$ and any set $\left\{s_{1}, \ldots, s_{u}=n\right\} \subseteq\{2, \ldots, n\}$, there exists a local Noetherian domain $B$ with maximal ideal $M$ and $M$-adic completion $\hat{B}$ such that $\left\{s_{1}, \ldots, s_{u}\right\}=\{v \mid$ there exists a saturated chain of prime ideals of length $v$ in $B$ between (0) and $M\}=\{v \mid$ there exists a minimal prime ideal of $\hat{B}$ of coheight $v\}$.

In $\$ 4$ we use our gluing process to construct a Noetherian domain $A$ with quotient field $L$ and a Noetherian domain $B$ contained between $A$ and $L$ such that: $A \hookrightarrow B$ possesses the Going Up and the Going Down properties, $A[X] \hookrightarrow B[X]$ is unibranched and $A[X] \hookrightarrow B[X]$ possesses neither the Going Up property nor the Going Down property. This gives a negative answer to several open questions.

In $\$ 5$ we construct a quasi local domain $D$ with maximal ideal $M$ that fails badly to satisfy the altitude formula: the height of $M$ is infinite, but the integral closure of $D$ has a height 1 prime ideal lying over $M$. 
In this paper all rings are commutative with identity, and a prime ideal is always different from the unit ideal. The symbol $\subseteq$ denotes inclusion and the symbol $\subset$ denotes proper inclusion. A totally ordered subset of an ordered set $\mathbb{Q}$ is a chain; a chain $\alpha_{0}<\alpha_{1}<\cdots<\alpha_{r}$ of $\mathbb{Q}$ is said to be saturated if for every $i=0,1, \ldots$, $r-1$ the elements $\alpha_{i}<\alpha_{i+1}$ are consecutive in $\mathcal{Q}$, i.e. there exists no element $\beta$ of $\mathbb{Q}$ such that $\alpha_{i}<\beta<\alpha_{i+1}$; in this case $r$ is the length of the chain. If $A$ is a ring, Spec $A$ denotes the set of the prime ideals of $A$ together with the order defined by the inclusion. A ring $A$ is catenarian if, given any prime ideals $P \subset Q$ of $A$, all the saturated chains of prime ideals between $P$ and $Q$ have the same length. Let $Q$ and $\mathbb{Q}^{\prime}$ be two ordered sets; $\psi: \mathbb{Q} \rightarrow \mathbb{Q}^{\prime}$ is an embedding if $\psi$ is injective and if given any elements $\alpha_{1}, \alpha_{2}$ of $\mathcal{Q}$ we have $\psi\left(\alpha_{1}\right)<\psi\left(\alpha_{2}\right)$ if and only if $\alpha_{1}<\alpha_{2}$. Let $A, B$ be two rings such that $A \subseteq B$. The extension $A \hookrightarrow B$ possesses the Lying Over property if given any prime ideal $P$ of $A$ there exists a prime ideal $P^{\prime}$ of $B$ such that $P^{\prime} \cap A=P . A \hookrightarrow B$ is unibranched if given any prime ideal $P$ of $A$ there exists a unique prime ideal $P^{\prime}$ of $B$ such that $P^{\prime} \cap A=P . A \hookrightarrow B$ possesses the Going $U p$ property if given any prime ideals $P \subset Q$ of $A$, and given any prime ideal $P^{\prime}$ of $B$ such that $P^{\prime} \cap A=P$, there exists a prime ideal $Q^{\prime}$ of $B$ such that $Q^{\prime} \supset P^{\prime}$ and $Q^{\prime} \cap A=Q \cdot A \hookrightarrow B$ possesses the Going Down property if given any prime ideals $P \subset Q$ of $A$ and given any prime ideal $Q^{\prime}$ of $B$ such that $Q^{\prime} \cap A=Q$, there exists a prime ideal $P^{\prime}$ of $B$ such that $P^{\prime} \subset Q^{\prime}$ and $P^{\prime} \cap A=P$.

2. A gluing process for maximal ideals. Let $K$ be an algebraically closed field, and $V$ be an affine variety over $K$ with $R$ as ring of regular functions. Let $P_{1}, \ldots, P_{s}$ be points of $V$ and glue them together to get a new point $P^{\prime}$, i.e. take $V^{\prime}=$ $V \backslash\left\{P_{1}, \ldots, P_{s}\right\} \cup\left\{P^{\prime}\right\}$. If we look for a structure of quotient affine variety on $V^{\prime}$, the most natural candidate to consider for the ring of regular functions of $V^{\prime}$ is

$$
D=\left\{f \in R \backslash f\left(P_{1}\right)=\cdots=f\left(P_{s}\right)\right\}=\left\{f \in R / \varepsilon_{1}(f)=\cdots=\varepsilon_{s}(f)\right\}
$$

where $\varepsilon_{i}: R \rightarrow K$ is the function evaluation at the point $P_{i}$. It turns out that indeed, via this ring $D, V^{\prime}$ has a structure of quotient affine variety. This geometric operation of gluing points is the motivation, as well as a special case, of the following process of "gluing" maximal ideals of a ring $R$. This process generalizes the technique used by Nagata in [11].

TheOREM A. Let $R$ be a ring, let $K_{1}, K_{2}, \ldots, K_{t}$ be fields and let $M_{11}, \ldots, M_{1 s_{1}}, M_{21}, \ldots, M_{2 s_{2}}, \ldots, M_{t 1}, \ldots, M_{t s_{t}}$ be maximal ideals of $R$. Let $\Lambda$ be the set $\cup_{i=1}^{t}\{i\} \times\left\{1, \ldots, s_{i}\right\}$; for every $(i, j) \in \Lambda$ let $\varepsilon_{i j}: R \rightarrow K_{i}$ be a surjective homomorphism with kernel $M_{i j}$. Let $D=\left\{f \in R \mid \varepsilon_{i 1}(f)=\cdots=\varepsilon_{i i_{i}}(f)\right.$ for $i=$ $1, \ldots, t\}$. Then:

(a) $R$ is a finite $D$-module, the conductor of $D$ in $R$ is $\cap_{(i, j) \in \Lambda} M_{i j}$, and for any $(i, j) \in \Lambda, \varepsilon_{i j}(D)$ is equal to $K_{i}$.

(b) For every $i \in\{1, \ldots, t\},\left\{M_{i 1}, \ldots, M_{i s_{i}}\right\}$ is the set of the prime ideals of $R$ that lie over $M_{i 1} \cap D$. For every $(i, j) \in \Lambda$, letting $\varphi_{i j}: R \rightarrow R / M_{i j}$ be the canonical homomorphism, $\varphi_{i j}(D)$ is equal to $R / M_{i j}$.

(c) If $P$ is a prime ideal of $R$ that does not belong to $\left\{M_{i j} \mid(i, j) \in \Lambda\right\}$ then $P$ is the only prime ideal of $R$ that lies over $P \cap D$. Furthermore, letting $\varphi_{P}: R \rightarrow R / P$ be the 
canonical homomorphism, the quotient field of $\varphi_{P}(D)$ is equal to the quotient field of $R / P$; if $P$ is not contained in any $M_{i j}$, then $\varphi_{P}(D)$ is even equal to $R / P$.

(d) If $P$ is a prime ideal of $R$ that does not belong to $\left\{M_{i j} \mid(i, j) \in \Lambda\right\}$ then $D_{P \cap D}=R_{P}$.

REMARK. If in Theorem A we take $t=1, K_{1}=K \subseteq R$, and if we suppose that $R$ is a finite $K$-algebra, then $D$ is a finite $K$-algebra too [1, Proposition 7.8, p. 81], and we get the geometric result mentioned before.

Proof. It is sufficient to prove the theorem with $t=1$ because by repeating $t$ times this weaker version, we will get the stronger version. In order to simplify the notations, we shall write $K$ instead of $K_{1}, M_{j}$ instead of $M_{1 j}, s$ instead of $s_{1}, \varepsilon_{j}$ instead of $\varepsilon_{1 j}, \varphi_{j}$ instead of $\varphi_{1 j}$.

(a) Let $\varepsilon: R \rightarrow K^{s}$ be the homomorphism defined by $\varepsilon(f)=\left(\varepsilon_{1}(f), \ldots, \varepsilon_{s}(f)\right)$. Since each $\varepsilon_{j}$ is surjective, and since the kernels of the $\varepsilon_{j}$ 's are pairwise comaximal, $\varepsilon$ is surjective by the Chinese Reminder Theorem. Note that by definition, $D$ is the inverse image of the diagonal of $K^{s}$. Let $f_{1}, \ldots, f_{s} \in R$ such that $\varepsilon\left(f_{1}\right)=$ $(1,0, \ldots, 0), \ldots, \varepsilon\left(f_{s}\right)=(0, \ldots, 0,1)$; we will show that $R=D+D f_{1}$ $+\cdots+D f_{s}$. Let $r \in R$; for $j=1, \ldots, s$, let $r_{j} \in R$ such that $\varepsilon\left(r_{j}\right)=$ $\left(\varepsilon_{j}(r), \ldots, \varepsilon_{j}(r)\right)$; clearly we have $r_{j} \in D$ and

$$
\begin{aligned}
\varepsilon(r)= & \left(\varepsilon_{1}(r), \ldots, \varepsilon_{s}(r)\right)=\left(\varepsilon_{1}(r), \ldots, \varepsilon_{1}(r)\right) \cdot(1,0, \ldots, 0)+\cdots \\
& +\left(\varepsilon_{s}(r), \ldots, \varepsilon_{s}(r)\right) \cdot(0, \ldots, 0,1) \\
= & \sum_{j=1}^{s} \varepsilon\left(r_{j}\right) \varepsilon\left(f_{j}\right)=\varepsilon\left(\sum_{j=1}^{s} r_{j} f_{j}\right),
\end{aligned}
$$

hence $r-\sum_{j=1}^{s} r_{j} f_{j} \in \operatorname{ker} \varepsilon=M_{1} \cap \cdots \cap M_{s} \subseteq D$; thus $R=D+D f_{1}$ $+\cdots+D f_{s}$. Now, it is clear that $\cap_{j=1}^{s} M_{j}$ is a common ideal of $D$ and $R$; furthermore, it will be checked in (b) that $M_{1}$ lies over $\cap_{j=1}^{s} M_{j}$ so that, $D \hookrightarrow R$ being an integral extension, $\bigcap_{j=1}^{s} M_{j}$ is a maximal ideal of $D$; thus $\cap_{j=1}^{s} M_{j}$ is the conductor of $D$ in $R$. Now, let $j \in\{1, \ldots, s\}$ and $\xi \in K$; let $d \in R$ such that $\varepsilon(d)=(\xi, \ldots, \xi)$; then, by definition itself we have $d \in D$ and $\varepsilon_{j}(d)=\xi$; thus $\varepsilon_{j}(D)=K$.

(b) Let $j \in\{1, \ldots, s\}$; we will show that $M_{j} \cap D=\cap_{k=1}^{s} M_{k}$. Let $\xi \in M_{j} \cap$ $D$; for every $k=1, \ldots, s$ we have $\varepsilon_{k}(\xi)=\varepsilon_{j}(\xi)=0$, hence $\xi \in \cap_{k=1}^{s} M_{k}$ and $M_{j} \cap D \subseteq \cap_{k=1}^{s} M_{k}$; since the other inclusion is clear, we get that $M_{j} \cap D=$ $\cap_{k=1}^{s} M_{k}$. Now if $M$ is any prime ideal of $R$ that lies over $\cap_{k=1}^{s} M_{k}$, we have $M \cap D=\cap_{k=1}^{s} M_{k}$, hence $M \supseteq \cap_{k=1}^{s} M_{k}$ and $M=M_{j}$ for some $j \in$ $\{1, \ldots, s\}$. Thus $\left\{M_{1}, \ldots, M_{s}\right\}$ is the set of prime ideals of $R$ that lies over $\cap_{k=1}^{s} M_{k}=M_{1} \cap D$. Now let $j \in\{1, \ldots, s\}, \varphi_{j}: R \rightarrow R / M_{j}$ the canonical homomorphism and $r \in R$; let $d \in R$ such that $\varepsilon(d)=\left(\varepsilon_{j}(r), \ldots, \varepsilon_{j}(r)\right)$; then, by definition itself we have $d \in D$ and $\varepsilon_{j}(d)=\varepsilon_{j}(r)$, hence $(r-d) \in \operatorname{ker} \varepsilon_{j}=M_{j}=$ $\operatorname{ker} \varphi_{j}$, i.e. $\varphi_{j}(d)=\varphi_{j}(r)$; thus $\varphi_{j}(D)=R / M_{j}$.

(c) Let $P$ be a prime ideal of $R$ that does not belong to $\left\{M_{j} \mid j=1, \ldots, s\right\}$. Let $P^{\prime} \neq P$ be any other prime ideal of $R$. If $P \subset P^{\prime}$, then $P \cap D \neq P^{\prime} \cap D$ since $R$ is integral over $D$. If $P^{\prime}=M_{j}$ for some $j \in\{1, \ldots, s\}$, then $P \cap D \neq P^{\prime} \cap D$ by 
(b). If $P \nsubseteq P^{\prime}$ and $P^{\prime} \neq M_{j} \forall j=1, \ldots, s$, then we can choose an element $d \in R$ such that $d \in P \cap\left(\cap_{j=1}^{s} M_{j}\right)$ and $d \notin P^{\prime}$; it is clear that such an element $d$ belongs to $P \cap D$ but not to $P^{\prime}$, so that $P \cap D \neq P^{\prime} \cap D$. Thus $P$ is the only prime ideal of $R$ that lies over $P \cap D$. Now let $\varphi_{P}: R \rightarrow R / P$ be the canonical homomorphism. If $P$ is not contained in $\cup_{j=1}^{s} M_{j}$, then $\cap_{j=1}^{s} M_{j}$ and $P$ are comaximal so that, by the Chinese Reminder Theorem, for every $r \in R$ there exists an element $d \in \cap_{j=1}^{s} M_{j}$ such that $d-r \in P$, i.e. an element $d \in D$ such that $\varphi_{P}(d)=\varphi_{P}(r)$; thus $\varphi_{P}(D)=R / P$. If $P$ is contained in $\cup_{j=1}^{s} M_{j}$, it is not true anymore that $\varphi_{P}(D)=R / P$, but it is still valid that the quotient field of $\varphi_{P}(D)$ is equal to the quotient field of $R / P$. Indeed, let $r \in R$; chcose $d \in \cap_{j=1}^{s} M_{j}$ such that $d \notin P$; then $d r \in D, \varphi_{P}(d)$ is a nonzero element of $\varphi_{P}(D)$ and $\varphi_{P}(d) \varphi_{P}(r)=$ $\varphi_{P}(d r) \in \varphi_{P}(D)$, i.e. $\varphi_{P}(r)$ belongs to the quotient field of $\varphi_{P}(D)$.

(d) Let $P$ be a prime ideal of $R$ that does not belong to $\left\{M_{j} \mid j=1, \ldots, s\right\}$, and let $S=D \backslash(P \cap D)$. Since $R$ is integral over $D$ and since $P$ is the only prime ideal of $R$ lying over $P \cap D$, we have $R_{P}=R_{S}$. Let $h: R \rightarrow R / \eta$ be the canonical homomorphism where $\eta=$ \{elements of $R$ that are annihilated by some element of $S$ \}; in order to show that $R_{S}=D_{S}$, it suffices to show that $R / \eta \subseteq(h(D))_{h(S)}$. Let $r \in R$; let $d \in \cap_{j=1}^{s} M_{j}$ such that $d \notin P$; we have $d \in D \backslash P \cap D=S$ and $d r \in D$, hence $h(d) h(r)=h(d r) \in h(D)$, i.e. $h(r) \in(h(D))_{h(S)}$.

3. On the spectrum of a noetherian ring. The main result of Heitmann in [5] is the following: given a finite ordered set $\mathscr{B}$, there exists a Noetherian domain $B$ and an embedding $\psi: \mathscr{B} \rightarrow$ Spec $B$ such that $\psi\left(\beta^{\prime}\right) \subset \psi\left(\beta^{\prime \prime}\right)$ are consecutive prime ideals of $B$ if $\beta^{\prime}<\beta^{\prime \prime}$ are consecutive elements of $\mathscr{B}$. In this section we prove a much stronger result that can also be considered as the analogue for Noetherian rings of the following result proved by Hochster [6, Proposition 10, p. 56] and Lewis [7, Theorem 2.10, p. 428]: every finite ordered set is order isomorphic to Spec $D$ for some ring $D$. As a corollary, we show that given any set $T$ of integers $>1$, there exists a local domain $B$ admitting $T$ as the set of coheights of the minimal prime ideals of the completion of $D$.

A Noetherian domain $D$ is regular if the localization $D_{M}$ is a regular local domain for every maximal ideal $M$ of $D$. It is well known that $D[X]$ is regular when $D$ is regular.

TheOrem B. Let $\mathscr{B}$ be a finite ordered set. Let $k$ be a field, $t_{1}, t_{2}, \ldots, t_{n}, \ldots$ an infinite set of indeterminates over $k$ and $K=k\left(t_{1}, \ldots, t_{n}, \ldots\right)$. Then, there exists $a$ reduced Noetherian ring $B$ and an embedding $\psi: \mathscr{B} \rightarrow \operatorname{Spec} B$ such that $(\mathscr{B}, B, \psi)$ satisfies the following conditions:

(i) $\psi$ establishes a bijection between the maximal (respectively minimal) elements of $\mathscr{B}$ and the maximal (resp. minimal) elements of Spec $B$.

(ii) For any elements $\beta^{\prime}, \beta^{\prime \prime} \in \mathscr{B}$ such that $\beta^{\prime}<\beta^{\prime \prime}$ is saturated in $\mathscr{B}$, the chain $\psi\left(\beta^{\prime}\right) \subset \psi\left(\beta^{\prime \prime}\right)$ is saturated in Spec $B$.

(iii) For any elements $\beta^{\prime}, \beta^{\prime \prime} \in \mathscr{B}$, there exists a saturated chain of prime ideals of length $r$ between $\psi\left(\beta^{\prime}\right)$ and $\psi\left(\beta^{\prime \prime}\right)$ (if and) only if there exists a saturated chain of length $r$ between $\beta^{\prime}$ and $\beta^{\prime \prime}$. 
(iv) For every $\beta \in \mathscr{B}$, the quotient field of $B / \psi(\beta)$ is isomorphic to $K$.

In the case that $\mathscr{B}$ has a least element, then the Noetherian domain $B$ can be chosen such that the following conditions are also satisfied:

(v) The integral closure $B^{\prime}$ of $B$ is a regular Noetherian domain.

(vi) If $\beta^{\prime}$ is any element of $\mathscr{B}$ and if $P^{\prime}$ is any prime ideal of $B^{\prime}$ lying over $\psi\left(\beta^{\prime}\right)$, then, for any element $\beta^{\prime \prime} \in \mathscr{B}, \beta^{\prime \prime} \geqslant \beta^{\prime}$, there exists a bijection $\tau$ : \{prime ideals $P^{\prime \prime}$ of $B^{\prime}$ such that $\left.P^{\prime \prime} \supseteq P^{\prime}, P^{\prime \prime} \cap B^{\prime}=\psi\left(\beta^{\prime \prime}\right)\right\} \rightarrow$ saturated chains between $\beta^{\prime}$ and $\left.\beta^{\prime \prime}\right\}$ such that height $\left(P^{\prime \prime} / P^{\prime}\right)=$ length $\tau\left(P^{\prime \prime}\right)$.

REMARK. The main reason for stating the conditions (iv)-(vi) is that they will be used as auxiliary results to prove conditions (ii) and (iii).

Proof. If $B$ has more than one minimal element, say $\alpha_{1}, \ldots, \alpha_{u}$, we can obviously embed $\mathscr{B}$ into an ordered set $\mathscr{B}^{\prime}$ having one more element that is its least element. Then, if $B^{\prime}$ is a Noetherian domain and $\psi^{\prime}: \mathscr{B}^{\prime} \rightarrow \operatorname{Spec} B^{\prime}$ is an embedding such that $\left(\mathscr{B}^{\prime}, B^{\prime}, \psi^{\prime}\right)$ satisfies (i)-(iv), it is clear that $B=$ $B^{\prime} /\left(\psi\left(\alpha_{1}\right) \cap \cdots \cap \psi\left(\alpha_{u}\right)\right)$ will be a reduced Noetherian ring and $\psi: \mathscr{B} \rightarrow$ Spec $B$ defined by $\psi(\beta)=\psi^{\prime}(\beta) /\left(\psi\left(\alpha_{1}\right) \cap \cdots \cap \psi\left(\alpha_{u}\right)\right)$ will be an embedding such that $(\mathscr{B}, B, \psi)$ satisfies (i)-(iv). Thus, from now on, we can suppose that $\mathscr{B}$ has a least element.

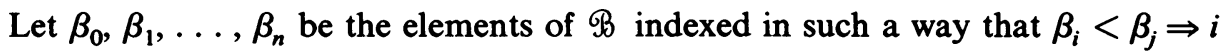
$<j$. For $i=0, \ldots, n$, let $\mathscr{B}_{i}=\left\{\beta_{0}, \ldots, \beta_{i}\right\}$. It is clear that, taking $B_{0}=K$ and $\psi_{0}: \mathscr{B}_{0} \rightarrow$ Spec $B_{0}$ defined by $\psi_{0}\left(\beta_{0}\right)=(0),\left(\mathscr{B}_{0}, B_{0}, \psi_{0}\right)$ satisfies (i)-(vi). Now, suppose that we have constructed a Noetherian domain $B_{i}$ and an embedding $\psi_{i}$ : $\mathscr{B}_{i} \rightarrow$ Spec $B_{i}$ such that $\left(\mathscr{B}_{i}, B_{i}, \psi_{i}\right)$ satisfies (i)-(vi) and consider $\mathscr{B}_{i+1}=\mathscr{B}_{i} \cup$ $\left\{\beta_{i+1}\right\}$. Let $\beta_{1}^{*}, \ldots, \beta_{s}^{*}$ be the immediate predecessors of $\beta_{i+1}$. Let $\mathcal{C}_{i+1}$ be the ordered set obtained in the following way: let $\gamma_{1}, \ldots, \gamma_{s}$ be distinct objects that do not belong to $\mathscr{B}$, take $\mathcal{C}_{i+1}=\mathscr{B}_{i} \cup\left\{\gamma_{1}, \ldots, \gamma_{s}\right\}$ with the order generated by the order on $\mathscr{B}_{i}$ and the relations $\beta_{j}^{*}<\gamma_{j}$ for $j=1, \ldots, s$.

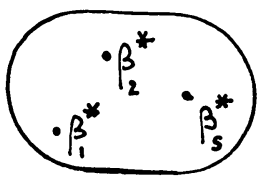

$\beta_{2}$

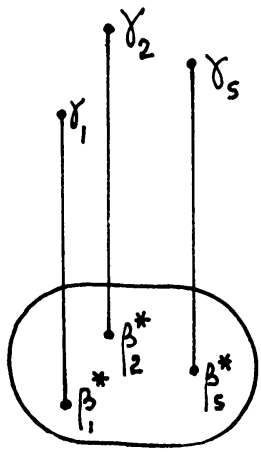

$e_{i+1}$

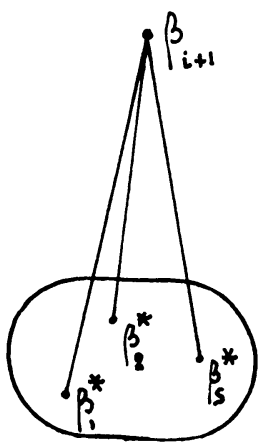

$B_{i+1}$

We will construct a Noetherian domain $B_{i+1}$ and an embedding $\psi_{i+1}: \mathscr{B}_{i+1} \rightarrow$ Spec $B_{i+1}$ such that $\left(\mathscr{B}_{i+1}, B_{i+1}, \psi_{i+1}\right)$ satisfies (i)-(vi) in two steps: first we will construct a Noetherian domain $C_{i+1}$ and an embedding $\rho_{i+1}: \bigcup_{i+1} \rightarrow \operatorname{Spec} C_{i+1}$ such that $\left(\mathcal{C}_{i+1}, C_{i+1}, \rho_{i+1}\right)$ satisfies those conditions, second we will glue together 
the prime ideals $\rho_{i+1}\left(\gamma_{1}\right), \ldots, \rho_{i+1}\left(\gamma_{s}\right)$ to get a new Noetherian domain $B_{i+1}$ and we will define the embedding $\psi_{i+1}: \mathscr{B}_{i+1} \rightarrow$ Spec $B_{i+1}$ in a natural way.

First step. When $D$ is a domain, we will denote its quotient field by $\operatorname{qf}(D)$. Consider $B_{i}[X]$ and let $\rho_{i+1}^{\prime}: \bigodot_{i+1} \rightarrow$ Spec $B_{i}[X]$ be defined by $\rho_{i+1}^{\prime}(\beta)=\psi_{i}(\beta)[X]$ for every $\beta \in \mathscr{B}_{i}$ and $\rho_{i+1}^{\prime}\left(\gamma_{j}\right)=\left(\psi_{i}\left(\beta_{j}^{*}\right), X\right)$ for $j=1, \ldots, s$. Let $\mu_{1}, \ldots, \mu_{t}$ be the maximal elements of $\mathcal{C}_{i+1}$; let $S=B_{i}[X] \backslash \cup \cup_{m=1}^{t} \rho_{i+1}^{\prime}\left(\mu_{m}\right)$, let $C_{i+1}=B_{i}[X]_{S}$ and let $\rho_{i+1}: \mathcal{C}_{i+1} \rightarrow \operatorname{Spec} B_{i}[X]_{S}$ be defined by $\rho_{i+1}(\delta)=\rho_{i+1}^{\prime}(\delta) B_{i}[X]_{S}$ for every $\delta \in \mathcal{C}_{i+1}$. It is clear that $B_{i}[X]$ and $B_{i}[X]_{S}$ are Noetherian domains and that $\rho_{i+1}^{\prime}$ and $\rho_{i+1}$ are embeddings that send the least element of $\mathcal{C}_{i+1}$ onto (0); in order to show that $\left(C_{i+1}, B_{i}[X]_{s}, \rho_{i+1}\right)$ satisfies (i)-(vi), it clearly suffices to show that $\left(\bigodot_{i+1}, B_{i}[X], \rho_{i+1}^{\prime}\right)$ satisfies (ii)-(vi). It is obvious that condition (ii) is satisfied. Now, for $\beta \in \mathcal{C}_{i+1} \backslash\left\{\gamma_{1}, \ldots, \gamma_{s}\right\}$, we have

$$
\mathrm{qf}\left(\frac{B_{i}[X]}{\rho_{i+1}^{\prime}(\beta)}\right)=\mathrm{qf}\left(\frac{B_{i}[X]}{\psi_{i}(\beta)[X]}\right) \simeq \mathrm{qf}\left(\frac{B_{i}}{\psi_{i}(\beta)}\right)(X) \simeq K(X) \simeq K ;
$$

for $j=1, \ldots, s$, we have

$$
\mathrm{qf}\left(\frac{B_{i}[X]}{\rho_{i+1}^{\prime}\left(\gamma_{j}\right)}\right)=\mathrm{qf}\left(\frac{B_{i}[X]}{\left(\psi_{i}\left(\beta_{j}^{*}\right), X\right)}\right) \simeq \mathrm{qf}\left(\frac{B_{i}}{\psi_{i}\left(\beta_{j}^{*}\right)}\right) \simeq K
$$

thus condition (iv) is satisfied. Let $B_{i}^{\prime}$ be the integral closure of $B_{i}$; then the integral closure of $B_{i}[X]$ is $B_{i}^{\prime}[X]$ which is a regular Noetherian domain; thus condition (v) is satisfied. Now, let us check that condition (vi) is satisfied. Let $\delta^{\prime}$ be any element of $\mathcal{C}_{i+1}$ and let $\mathcal{P}^{\prime}$ be any prime ideal of $B_{i}^{\prime}[X]$ lying over $\rho_{i+1}^{\prime}\left(\delta^{\prime}\right)$. If $\delta^{\prime}$ is a maximal element of $\bigodot_{i+1}$, i.e. if $\delta^{\prime}=\gamma_{j}$ for some $j \in\{1, \ldots, s\}$, then $\rho_{i+1}^{\prime}\left(\delta^{\prime}\right)=$ $\left(\psi_{i}\left(\beta_{j}^{*}\right), X\right)$ is a maximal ideal of $B_{i}[X]$ and condition (vi) is clearly satisfied. If $\delta^{\prime}$ is not a maximal element of $\mathcal{C}_{i+1}$, then $\delta^{\prime} \in \mathscr{B}_{i}$ and $\mathcal{P}^{\prime}$ must be of the type $P^{\prime}[X]$ with $P^{\prime}$ a prime ideal of $B_{i}^{\prime}$ lying over $\psi_{i}\left(\delta^{\prime}\right)$. Let $\delta^{\prime \prime} \geqslant \delta^{\prime}$ be any other element of $\mathcal{C}_{i+1}$ bigger than or equal to $\delta^{\prime}$. If $\delta^{\prime \prime}$ belongs to $\mathscr{B}_{i}$ also, then any prime ideal $\mathscr{P}^{\prime \prime}$ of $B_{i}^{\prime}[X]$ lying over $\rho_{i+1}^{\prime}\left(\delta^{\prime \prime}\right)=\psi_{i}\left(\delta^{\prime \prime}\right)[X]$ must be of the type $P^{\prime \prime}[X]$ with $P^{\prime \prime}$ a prime ideal of $B_{i}^{\prime}$ lying over $\psi_{i}\left(\delta^{\prime \prime}\right)$; then, it is clear that the application (prime ideals $\mathcal{P}^{\prime \prime}$ of $B_{i}^{\prime}[X]$ such that $\left.\mathscr{P} " \supseteq \mathscr{P}^{\prime}, \mathcal{P}^{\prime \prime} \cap B_{i}[X]=\psi_{i}\left(\delta^{\prime \prime}\right)[X]=\rho_{i+1}^{\prime}\left(\delta^{\prime \prime}\right)\right\} \rightarrow$ \{prime ideals $P^{\prime \prime}$ of $B_{i}^{\prime}$ such that $P^{\prime \prime} \supseteq P^{\prime}, P^{\prime \prime} \cap B_{i}=\psi_{i}\left(\delta^{\prime \prime}\right)$ \} defined by $\mathscr{P} " \leadsto$ $\mathscr{P}^{\prime \prime} \cap B_{i}^{\prime}$ is a bijection, and if we compose it with the bijection $\tau_{i}$ : \{prime ideals $P^{\prime \prime}$ of $B_{i}^{\prime}$ such that $\left.P^{\prime \prime} \supseteq P^{\prime}, P^{\prime \prime} \cap B_{i}=\psi_{i}\left(\delta^{\prime \prime}\right)\right\} \rightarrow$ saturated chains between $\delta^{\prime}$ and $\delta^{\prime \prime}$ \} given by the hypothesis of induction, we obtain a bijection $\tau_{i+1}^{\prime}$ : \{prime ideals $\mathscr{P}^{\prime \prime}$ of $B_{i}^{\prime}[X]$ such that $\left.\mathcal{P}^{\prime \prime} \supseteq \mathcal{P}^{\prime}, \mathscr{P}^{\prime \prime} \cap B_{i}[X]=\rho_{i+1}^{\prime}\left(\delta^{\prime \prime}\right)\right\} \rightarrow$ saturated chains between $\delta^{\prime}$ and $\delta^{\prime \prime}$ \} such that

$$
\text { height } \frac{\mathscr{P}^{\prime \prime}}{\mathscr{P}^{\prime}}=\text { height } \frac{P^{\prime \prime}[X]}{P^{\prime}[X]}=\text { height } \frac{P^{\prime \prime}}{P^{\prime}}=\text { length } \tau_{i}\left(P^{\prime \prime}\right)=\text { length } \tau_{i+1}^{\prime}\left(\mathscr{P}^{\prime \prime}\right) \text {. }
$$

If $\delta^{\prime \prime}$ does not belong to $\mathscr{B}_{i}$, then $\delta^{\prime \prime}=\gamma_{j}$ for some $j \in\{1, \ldots, s\}, \beta_{j}^{*}$ is the unique immediate predecessor of $\delta^{\prime \prime}$, and any prime ideal $\mathcal{P}^{\prime \prime}$ of $B_{i}^{\prime}[X]$ lying over $\rho_{i+1}^{\prime}\left(\delta^{\prime \prime}\right)=\left(\psi_{i}\left(\beta_{j}^{*}\right), X\right)$ must be of type $\left(P^{\prime \prime}, X\right)$ with $P^{\prime \prime}$ a prime ideal of $B_{i}^{\prime}$ lying over $\psi_{i}\left(\beta_{j}^{*}\right)$; then it is clear that the application (prime ideals $\mathscr{P}$ " of $B_{i}^{\prime}[X]$ such 
that $\left.\mathscr{P}^{\prime \prime} \supseteq \mathscr{P}^{\prime}, \mathscr{P}^{\prime \prime} \cap B_{i}[X]=\left(\psi_{i}\left(\beta_{j}^{*}\right), X\right)=\rho_{i+1}^{\prime}\left(\delta^{\prime \prime}\right)\right\} \rightarrow$ prime ideals $P^{\prime \prime}$ of $B_{i}^{\prime}$ such that $\left.P^{\prime \prime} \supseteq P^{\prime}, P^{\prime \prime} \cap B_{i}=\psi_{i}\left(\beta_{j}^{*}\right)\right\}$ defined by $\mathscr{P}^{\prime \prime} \leadsto \mathcal{P}^{\prime \prime} \cap B_{i}^{\prime}$ is a bijection; if we compose it with \{prime ideals $P^{\prime \prime}$ of $B_{i}^{\prime}$ such that $P^{\prime \prime} \supseteq P^{\prime}, P^{\prime \prime} \cap B_{i}=\psi_{i}\left(\beta_{j}^{*}\right)$ \} $\rightarrow$ ssaturated chains between $\beta^{\prime}$ and $\left.\beta_{j}^{*}\right\} \rightarrow$ saturated chains between $\beta^{\prime}$ and $\delta^{\prime \prime}$ \} where the first arrow represents the bijection $\tau_{i}$ given by the hypothesis of induction and the second arrow represents the bijection defined by $\left(\beta^{\prime}<\cdots<\right.$ $\left.\beta_{j}^{*}\right) \leadsto\left(\beta^{\prime}<\cdots<\beta_{j}^{*}<\delta^{\prime \prime}\right)$, we obtain a bijection $\tau_{i+1}^{\prime}$ : \{prime ideals $\mathscr{P}^{\prime \prime}$ of $B_{i}^{\prime}[X]$ such that $\left.\mathscr{P}^{\prime \prime} \supseteq \mathcal{P}^{\prime}, \mathscr{P}^{\prime \prime} \cap B_{i}[X]=\rho_{i+1}^{\prime}\left(\delta^{\prime \prime}\right)\right\} \rightarrow$ saturated chains between $\delta^{\prime}$ and $\delta^{\prime \prime}$ \} such that

$$
\text { height } \begin{aligned}
\frac{\mathscr{P}^{\prime \prime}}{\mathscr{P}^{\prime}} & =\text { height } \frac{\left(P^{\prime \prime}, X\right)}{P^{\prime}[X]}=\left(\text { height } \frac{P^{\prime \prime}}{P^{\prime}}\right)+1 \\
& =\text { length } \tau_{i}\left(P^{\prime \prime}\right)+1 \\
& =\text { length } \tau_{i+1}^{\prime}\left(\mathscr{P}^{\prime \prime}\right) .
\end{aligned}
$$

Thus condition (vi) is satisfied. Finally, let us check that condition (iii) is also satisfied. Let $\delta^{\prime}$ and $\delta^{\prime \prime}$ be two elements of $\mathcal{C}_{i+1}$ and let $\rho_{i+1}^{\prime}\left(\delta^{\prime}\right)=\mathscr{P}_{0} \subset \mathscr{P}_{1}$ $\subset \cdots \subset \mathscr{P}_{r}=\rho_{i+1}^{\prime}\left(\delta^{\prime \prime}\right)$ be a saturated chain of prime ideals in $B_{i}[X]$ of length $r>0$ between $\rho_{i+1}^{\prime}\left(\delta^{\prime}\right)$ and $\rho_{i+1}^{\prime}\left(\delta^{\prime \prime}\right)$. By the Going Up theorem, we can get a saturated chain of prime ideals $\mathscr{P}_{0}^{\prime} \subset \mathscr{P}_{1}^{\prime} \subset \cdots \subset \mathscr{P}_{r}^{\prime}$ in $B_{i}^{\prime}[X]$ such that $\mathscr{P}_{m}=$ $\mathscr{P}_{m}^{\prime} \cap B_{i}[X]$ for every $m=0, \ldots, r$. Since $B_{i}^{\prime}[X]$ is regular, it is catenarian $[12$, (34.8), p. 125] and we have height $\left(\mathscr{P}_{r}^{\prime} / \mathscr{P}_{0}^{\prime}\right)=r$; then, by condition (vi), there exists a saturated chain of length $r$ between $\delta^{\prime}$ and $\delta^{\prime \prime}$.

Second step. Set $C_{i+1}=B_{i}[X]_{s}$. Note that $\gamma_{1}, \ldots, \gamma_{s}$ are maximal elements of $\mathcal{C}_{i+1}$, and therefore that $\rho_{i+1}\left(\gamma_{1}\right), \ldots, \rho_{i+1}\left(\gamma_{s}\right)$ are maximal ideals of $C_{i+1}$. For every $j=1, \ldots, s, C_{i+1} / \rho_{i+1}\left(\gamma_{j}\right)$ being isomorphic to $K$ by condition (iv), we can choose a surjective homomorphism $\varepsilon_{j}: C_{i+1} \rightarrow K$ with kernel $\rho_{i+1}\left(\gamma_{j}\right)$. Let $B_{i+1}=\{f$ $\left.\in \mathcal{C}_{i+1} \mid \varepsilon_{1}(f)=\cdots=\varepsilon_{s}(f)\right\}$ and let $\psi_{i+1}: \mathscr{B}_{i+1} \rightarrow$ Spec $B_{i+1}$ be defined by $\psi_{i+1}(\beta)=\rho_{i+1}(\beta) \cap B_{i+1}$ for $\beta \neq \beta_{i+1}$ and $\psi_{i+1}\left(\beta_{i+1}\right)=\rho_{i+1}\left(\gamma_{1}\right) \cap B_{i+1}$. By part (a) of Theorem A, $C_{i+1}$ is a finite $B_{i+1}$-module; then, $C_{i+1}$ being Noetherian, $B_{i+1}$ is Noetherian [4, Theorem 2, p. 281]. We know that $\left(\mathcal{C}_{i+1}, C_{i+1}, \rho_{i+1}\right)$ satisfies (i)-(vi); using parts (b) and (c) of Theorem A, it is merely routine to check that $\left(\Re_{i+1}, B_{i+1}, \psi_{i+1}\right)$ satisfies (i), (ii), (iv)-(vi). Now, we check that it satisfies condition (iii) also. Let $\beta^{\prime}$ and $\beta^{\prime \prime}$ be two elements of $\mathscr{B}_{i+1}$ and let $\psi_{i+1}\left(\beta^{\prime}\right)=\mathscr{P}_{0} \subset \mathscr{P}_{1}$ $\subset \cdots \subset \mathscr{P}_{r}=\psi_{i+1}\left(\beta^{\prime \prime}\right)$ be a saturated chain of prime ideals of length $r>0$ between $\psi_{i+1}\left(\beta^{\prime}\right)$ and $\psi_{i+1}\left(\beta^{\prime \prime}\right)$. By the Going Up theorem, we can get a saturated chain of prime ideals $\mathscr{P}_{0}^{\prime} \subset \mathscr{P}_{1}^{\prime} \subset \cdots \subset \mathscr{P}_{r}^{\prime}$ in $B_{i+1}^{\prime}$ such that $\mathscr{P}_{m}=\mathscr{P}_{m}^{\prime} \cap B_{i+1}$ for every $m=0, \ldots, r$. Since $B_{i+1}^{\prime}$ is regular, it is catenarian [12, (34.8), p. 125] and we have height $\left(\mathscr{P}_{r}^{\prime} / \mathscr{P}_{0}^{\prime}\right)=r$; then, by condition (vi) there exists a saturated chain of length $r$ between $\delta^{\prime}$ and $\delta^{\prime \prime}$.

The following corollary strengthens a result of Doering [3, Example 2.5].

COROllary. Let $n \geqslant 2$ be an integer and let $T=\left\{t_{1}, \ldots, t_{u-1}, n\right\}$ be a subset of $\{2, \ldots, n\}$ containing $n$. Then, there exists a local domain $B$ with maximal ideal $M$ and $M$-adic completion $\hat{B}$ such that $T=\{r \mid$ there exists a saturated chain of prime ideals of length $r$ in $B$ between (0) and $M\}=\{r \mid$ there exists a minimal prime ideal of $\hat{\boldsymbol{B}}$ of coheight $\boldsymbol{r}$ \}. 
Proof. Let $\mathscr{B}$ be an ordered set that has a least element $\alpha$, a greatest element $\beta$ and has exactly $u$ saturated chains between $\alpha$ and $\beta$, of length $t_{1}, \ldots, t_{u-1}, n$ respectively. According to Theorem $B$, there exists a local domain $B$ and an embedding $\psi: \mathscr{B} \rightarrow$ Spec $B$ such that $(\mathscr{B}, B, \psi)$ satisfies (i)-(vi). In particular we have by (iii) that $T=\{r \mid$ there exists a saturated chain of prime ideals of length $r$ between (0) and $M\}$. Now, by [13, Theorem 2.14, p. 111] we know that $\{r \mid$ there exists a minimal prime ideal of $\hat{B}$ of coheight $r\}=\{r \mid$ there exists a saturated chain of prime ideals of length $(r+1)$ in $B[X]$ between $(0)$ and $(M, X)\}$. On the other hand, we have seen in the first step of the proof of Theorem B that conditions (v) and (vi) imply $\{r \mid$ there exists a saturated chain of prime ideals of length $(r+1)$ in $B[X]$ between $(0)$ and $(M, X)\} \subseteq T$; since the other inclusion is clear, we get the equality. Then, we obtain that $T=\{r \mid$ there exists a minimal prime ideal of $\hat{B}$ of coheight $r$.

4. Going Up and Going Down in polynomial rings. Let $A$ be a domain and $L$ its quotient field; let $B$ be an overring of $A$, i.e. a ring such that $A \subseteq B \subseteq L$. We consider the following three open questions:

Question 1 [8, p. 706]. If the extension $A \hookrightarrow B$ possesses the Going Up property, then does the extension $A[X] \hookrightarrow B[X]$ possess it also?

Note that when $B$ is contained in the integral closure of $A$, then the answer to Question 1 is clearly YES since, in this case, $A[X] \hookrightarrow B[X]$ is an integral extension.

Related to that Question 1, we have:

Question 2. If the extension $A \hookrightarrow B$ possesses the Going Up property, and if the extension $A[X] \hookrightarrow B[X]$ is unibranched, then does the extension $A[X] \hookrightarrow B[X]$ possess the Going Up property?

The third question is related to the property of Going Down:

Question 3 [2, p. 180]. If the extension $A \hookrightarrow B$ possesses the property of Going Down, and if the extension $A[X] \hookrightarrow B[X]$ is unibranched, then does the extension $A[X] \hookrightarrow B[X]$ possess the Going Down property?

Note that when $B$ is contained in the integral closure of $A$, then McAdam has given an affirmative answer to Question 3 [9, Theorem 3, p. 635]. Note also that using a result of Kaplansky [8, Theorem A, p. 704] it is easy to show the existence of an extension $A \hookrightarrow B$ such that: $B$ is contained in the integral closure of $A$, $A \hookrightarrow B$ possesses the Going Down property, $A[X] \hookrightarrow B[X]$ does not possess the Going Down property.

Observe that the analogous questions for the Lying Over property has an affirmative answer; indeed we have:

Proposition [8, Proposition 1, P. 708]. If the extension $A \hookrightarrow B$ possesses the Lying Over property, then the extension $A[X] \hookrightarrow B[X]$ possesses it also.

Now we show that in general the answer to the three previous questions is negative, even when $A$ and $B$ are Noetherian. Given any integer $n>1$, we will construct an $n$-dimensional Noetherian domain $A$ with quotient field $L$ and an $n$-dimensional Noetherian domain $B$ that is contained between $A$ and $L$ such that: $A \hookrightarrow B$ possesses the properties of Going Up and Going Down, $A[X] \hookrightarrow B[X]$ in 
unibranched, $A[X] \hookrightarrow B[X]$ possesses neither the Going Up property nor the Going Down property.

ExAmple $C$. Let $k$ be a field, let $t_{1}, \ldots, t_{n}, \ldots$ be an infinite set of indeterminates over $k$ and let $K=k\left(t_{1}, \ldots, t_{n}, \ldots\right)$. Let $n>1$ be an integer and let $X, Y_{1}, \ldots, Y_{n}$ be indeterminates over $K$. Let $S=K\left[X, Y_{1}, \ldots, Y_{n}\right] \backslash(X) \cup$ $\left(Y_{1}, \ldots, Y_{n}\right)$ and $R=K\left[X, Y_{1}, \ldots, Y_{n}\right]_{s}$. Let $M_{1}=X R$ and $M_{2}=$ $\left(Y_{1}, \ldots, Y_{n}\right) R$ be the maximal ideals of $R$. For every $i \in\{1,2\}$, it is clear that $R / M_{i}$ is isomorphic to $K$, hence that we can choose a surjective homomorphism $\varepsilon_{i}$ : $R \rightarrow K$ such that $\operatorname{ker} \varepsilon_{i}=M_{i}$. Let $A=\left\{x \in R \mid \varepsilon_{1}(x)=\varepsilon_{2}(x)\right\}$ and $B=R_{M_{2}}$; we will show $A \hookrightarrow B$ is an example of what we announced.

$R$ is Noetherian, hence $B=R_{M_{2}}$ is Noetherian; $R$ is a finite $A$-module by part (a) of Theorem A, hence $A$ is Noetherian [4, Theorem 2, p. 281]. Also by part (a) of Theorem $A, B$ is contained in the quotient field of $B$. Finally it is obvious that both $A$ and $B$ are $n$-dimensional.

Since $A \hookrightarrow R$ is integral, $A \hookrightarrow R$ possesses the Going Up property. Now, by parts (b) and (c) of Theorem A, we know the following: the domain $A$ has only one maximal ideal $M, M_{1}$ and $M_{2}$ are exactly the prime ideals of $R$ that lie over $M$, and above any prime ideal $P \neq M$ of $A$ lies exactly one prime ideal of $R$. Since height $M_{1}=1$, we can conclude that the extension $A \hookrightarrow R_{M_{2}}=B$ is unibranched and possesses the Going Up property; consequently, it also possesses the Going Down property.

We already know that $A \hookrightarrow R_{M_{2}}=B$ is unibranched. But again by parts (b) and (c) of Theorem A, we also know that if $P^{\prime}$ is any prime ideal of $B$ and if $\varphi$ : $B \rightarrow B / P^{\prime}$ is the canonical homomorphism, then we have $\operatorname{qf}(\varphi(A))=\operatorname{qf}\left(B / P^{\prime}\right)$. From that, we can conclude that $A[X] \hookrightarrow B[X]$ is unibranched [8, Theorem 3, p. 708].

Finally we will show that $A[X] \hookrightarrow B[X]$ does not possess the Going Up property; being unibranched it will not possess the Going Down property either. Let $x \in$ $M_{1} \backslash M_{2}$; then $(X-x) R[X]$ is a prime ideal of $R[X]$ properly contained in $\left(M_{1}, X\right)$, and $(X-x) R[X] \cap A[X]$ is a prime ideal of $A[X]$ properly contained in $\left(M_{1}, X\right) \cap A[X]=(M, X)$. Let $P$ be the only prime ideal of $B[X]$ that lies over $(X-x) R[X] \cap A|X|$ (clearly $P$ has to be $(X-x) B[X]$ ); we claim that $P$ is not contained in the only prime ideal of $B[X]$ that lies over $(M, X)$, i.e. that $P$ is not contained in $\left(M_{2} B, X\right)$. Indeed, if it did, $X-x$ would belong to $\left(M_{2} B, X\right)$, hence $x$ would belong to $M_{2} B \cap R=M_{2}$, which is absurd. Thus, $A[X] \hookrightarrow B[X]$ possesses neither the Going Up property nor the Going Down property.

Remark. With $n \geqslant 2$, the domains $A$ and $B$ constructed in Example $\mathrm{C}$ are such that $A[X] \hookrightarrow B[X]$ is unibranched, $B[X]$ is catenarian, $A[X]$ is not catenarian.

5. An extravagant example. In this section we construct a quasi local domain $D$ with maximal ideal $M$ that fails to satisfy the altitude formula in a very extreme way: the height of $M$ is infinite, but the integral closure of $D$ has a height 1 prime ideal that lies over $M$.

EXAMPLE D. Let $k$ be a field and let $t_{1}, \ldots, t_{n}, \ldots$ be indeterminates over $k$. Let $K=k\left(t_{1}, \ldots, t_{n}, \ldots\right)$ and let $X, Y_{1}, \ldots, Y_{n}, \ldots$ be indeterminates over $K$. Let 


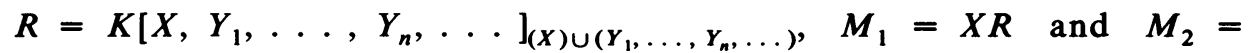
$\left(Y_{1}, \ldots, Y_{n}, \ldots\right) R$. It is clear that $R / M_{1}$ and $R / M_{2}$ are both isomorphic to $K$; let $\varepsilon_{1}: R \rightarrow K$ and $\varepsilon_{2}: R \rightarrow K$ be surjective homomorphisms such that ker $\varepsilon_{1}=M_{1}$ and $\operatorname{ker} \varepsilon_{2}=M_{2}$; let $D=\left\{x \in R \mid \varepsilon_{1}(x)=\varepsilon_{2}(x)\right\}$. $R$ is integrally closed; then, by Theorem A, $R$ is the integral closure of $D$; furthermore, $M_{1} \cap M_{2}$ is the only maximal ideal of $D$, and the prime ideals of $R$ that lie over it are $M_{1}$ and $M_{2}$. It is clear that we have height $M_{1}=1$ and height $M_{1} \cap M_{2}=$ height $M_{2}=\infty$.

We wish to acknowledge the enlightening conversations we had with Karl Otto Stöhr during the preparation of this paper.

\section{REFERENCES}

1. M. Atiyah and I. MacDonald, Introduction to commutative algebra, Addison-Wesley, Reading, Mass., 1969.

2. J. Dawson and D. E. Dobbs, On Going Down in polynomial rings, Canad. J. Math. 26 (1974), 177-184.

3. A. M. S. Doering, Chains of prime ideals in Noetherian domains, J. Pure Appl. Algebra (to appear).

4. P. Eakin, The converse to a well-known theorem on Noetherian rings, Math. Ann. 177 (1968), 278-282.

5. R. Heitmann, Examples of noncatenary rings, Trans. Amer. Math. Soc. 247 (1979), 125-136.

6. M. Hochster, Prime ideal structure in commutative rings, Trans. Amer. Math. Soc. 142 (1969), 43-60.

7. W. Lewis, The spectrum of a ring as a partially ordered set, J. Algebra 25 (1973), 419-434.

8. S. McAdam, Going Down in polynomial rings, Canad. J. Math. 23 (1971), 704-711.

9. , Going Down, Duke Math. J. 39 (1972), 633-636.

10. Saturated chains in Noetherian rings, Indiana Univ. Math. J. 23 (1974), 719-728.

11. M. Nagata, On the chain problem of prime ideals, Nagoya Math. J. 10 (1956), 51-64.

12. __ Local rings, Interscience, New York, 1962.

13. L. Ratliff and S. McAdam, Maximal chains of prime ideals in integral extension domains. I, Trans. Amer. Math. Soc. 224 (1976), 103-116.

instituto de Matematica, Universidade Federal do Rio Grande do Sul, Rua Sarmento Lette 425, 90.000 Porto Alegre, Brazil

Instituto de Matematica Pura e Aplicada, Rua luis de Camões 68, 20060 Rio de Janeiro, BRAZIL 